\title{
Phenomenology of black hole evaporation with a cosmological constant
}

\section{J. Labbé, A. Barrau*, J. Grain}

Laboratory for Subatomic Physics and Cosmology

CNRS-IN2P3, Joseph Fourier University, France

E-mail: Aurelien.Barraudcern. ch

In this brief note, we investigate some possible experimental consequences of the de-Sitter or Anti-de-Sitter background spacetime structure for $d$-dimensional evaporating black holes. Possible observational signatures in Large Hadron Collider (LHC) events are considered in the framework of the Arkani-Hamed-Dimopoulos-Dvali (ADD) braneworld model. Lower bounds on the value of the bulk cosmological constant required to produce visible effects are derived thanks to a dynamical Monte-Carlo simulation. This preliminary study has to be refined by the accurate computation of the greybody factors. It opens a new way to investigate the structure of nonasymptotically flat higher-dimensional spacetimes.

International Europhysics Conference on High Energy Physics

July 21st - 27th 2005

Lisboa, Portugal

${ }^{*}$ Speaker. 
It has recently been shown (see [1] for a review) that the fundamental structure of the background spacetime modifies the shape of the Hawking spectrum for evaporating black holes. This has been extensively studied to determine the number of extra dimensions [2]. The rotation of the black hole [3] or a modification of the gravitational theory with higher order curvature corrections [4] may also change the characteristics of the emitted spectrum. In this note, we investigate the possible consequences of a $d$-dimensional cosmological constant. To study this question into the details, the greybody factors are required. They have been computed for scalar fields in a Schwarzshild-de-Sitter spacetime [5] (available at this URL : http://psc.in2p3.fr/ams/greybody/) but they are not yet known for fermions, gauge bosons, and for a negative cosmological constant [6]. As a first attempt in this direction which aims at giving rough orders of magnitude, the present work does not take into account the accurate greybody factors and only deals with the consequences of the cosmological constant on the temperature.

Following [7], we consider the possible creation of black holes at colliders in the framework of the ADD model which allows for a low fundamental Planck scale $M_{d}$ related to the apparent 4-dimensional Planck scale $M_{P l}$ through the volume of the compactified dimensions, $V_{d-4}$, via $M_{d}=\left(M_{P l}^{2} / V_{d-4}\right)^{1 /(d-2)}$. We now consider that the background spacetime is described by a nonzero cosmological constant $\Lambda$ (which, in $d$ dimension, is not directly constrained by the tiny value measured by cosmological observations in 4 dimensions). The metric can be written as $d s^{2}=$ $g_{\mu \nu} d x^{\mu} d x^{v}=f(r) d t^{2}-d r^{2} / f(r)-r^{2} d \Omega_{d-2}^{2}$ where $d \Omega_{d-2}$ is the solid angle and $g_{00}=f(r)=1-$ $\frac{16 \pi G M}{(d-2) A_{d-2} r^{d-3}}-\frac{2 \Lambda r^{2}}{(d-1)(d-2)} . G=1 / M_{d}^{d-2}$ is the $d$-dimensional gravity constant and $A_{d-2}=\frac{2 \pi^{(d-1) / 2}}{\Gamma((d-1) / 2)}$ is the area of a unit $(d-2)$ sphere. Figure 1 shows the behavior of $g_{00}$ for different values of the cosmological constant. The temperature of the black hole, and the relation between its radius $r_{H}$
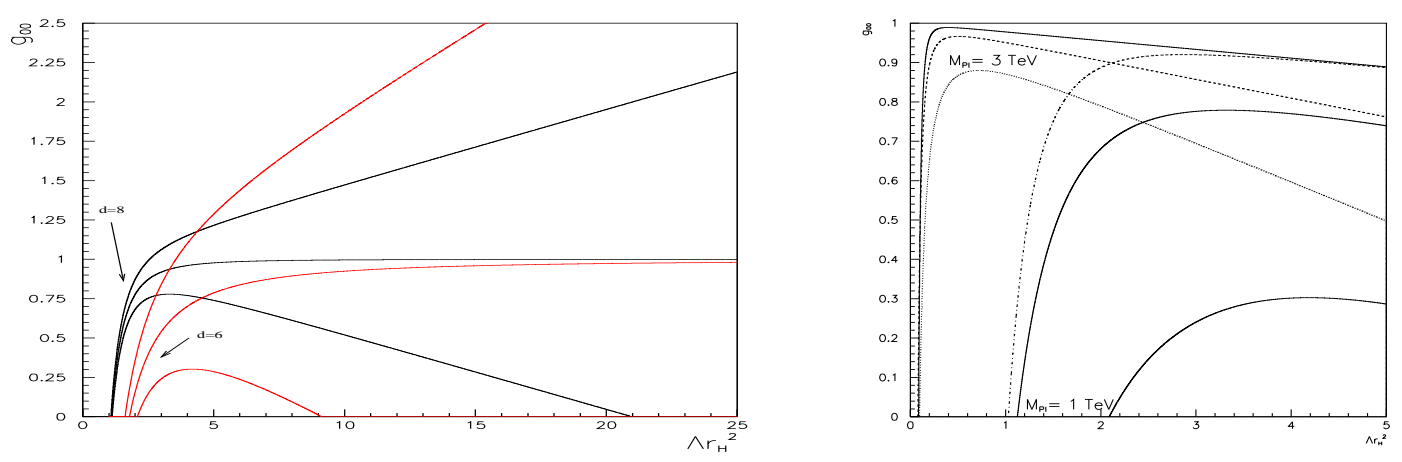

Figure 1: Metric element $g_{00}=f\left(r_{H}\right)$ for a black hole with mass $5 \mathrm{TeV}$. Left : $M_{d}=1 \mathrm{TeV}$ and (from the bottom curve to the upper one) $\Lambda=1,0$ and $-1 \mathrm{TeV}^{2}$ for 6 and 8 dimensions. Right : $\Lambda=1 \mathrm{TeV}^{2}$ for two Planck scales : 1 and $3 \mathrm{TeV}$ and (from the bottom curve to the upper one) $d=6,8,11$.

and mass $M$ are given by :

$$
T=\frac{d-3}{4 \pi r_{H}}\left[1-\frac{2 \Lambda r_{H}^{2}}{(d-3)(d-2)}\right], M=\frac{(d-2) A_{d-2} r_{H}^{n+1}}{16 \pi G}\left[1-\frac{2 \Lambda r_{H}^{2}}{(d-3)(d-2)}\right]
$$

The consequences of the cosmological constant are more important when the number of dimensions is smaller. For $\Lambda>0$, there is a maximum radius $r_{\max }=\sqrt{(d-3)(d-2) / 2 \Lambda}$ corre- 
sponding to a vanishing temperature when the horizon of the black hole coincide with the cosmological horizon.

With $\Lambda=0$ and $M_{d}=1 \mathrm{TeV}$, the temperature of a black hole possibly formed at the LHC varies from $0.18 \mathrm{TeV}$ for $d=6$ to $0.63 \mathrm{TeV}$ for $d=11$ (for $M=5 \mathrm{TeV}$ ). The modifications induced by $\Lambda$, together with the maximum mass value, are given in Fig. 2. It shows the order of magnitude of a cosmological constant that could be probed at colliders.
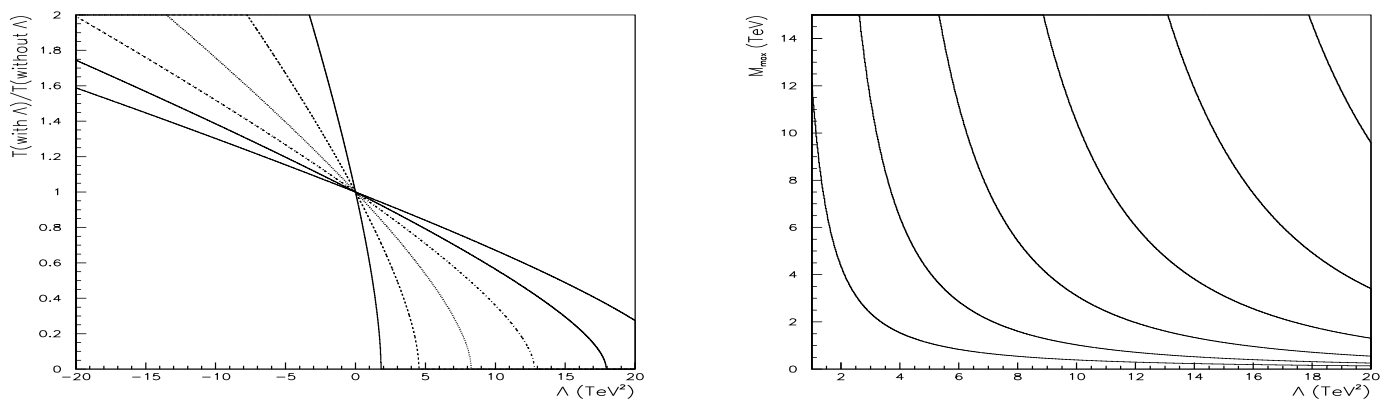

Figure 2: Left : ratio of the temperature with a cosmological constant to the temperature without a cosmological constant for a $5 \mathrm{TeV}$ black hole. Right : Maximum mass for $\Lambda=1 \mathrm{TeV}^{2}$. Both plots correspond to a Planck scale fixed at $1 \mathrm{TeV}$ and to $d$ between 6 and 11 .

In Fig. 3 (left), the observable flux is shown, as obtained by integrating over time the Hawking spectrum with a modified temperature (under the hypothesis of a thermalized emission). The effects of the cosmological constant are easier to measure with a sudden evaporation hypothesis, as can be understood from Fig. 3 (right), as it corresponds to a larger average radius leading to a highest sensitivity to $\Lambda$ (the curvature induced by the black-hole being relatively smaller).
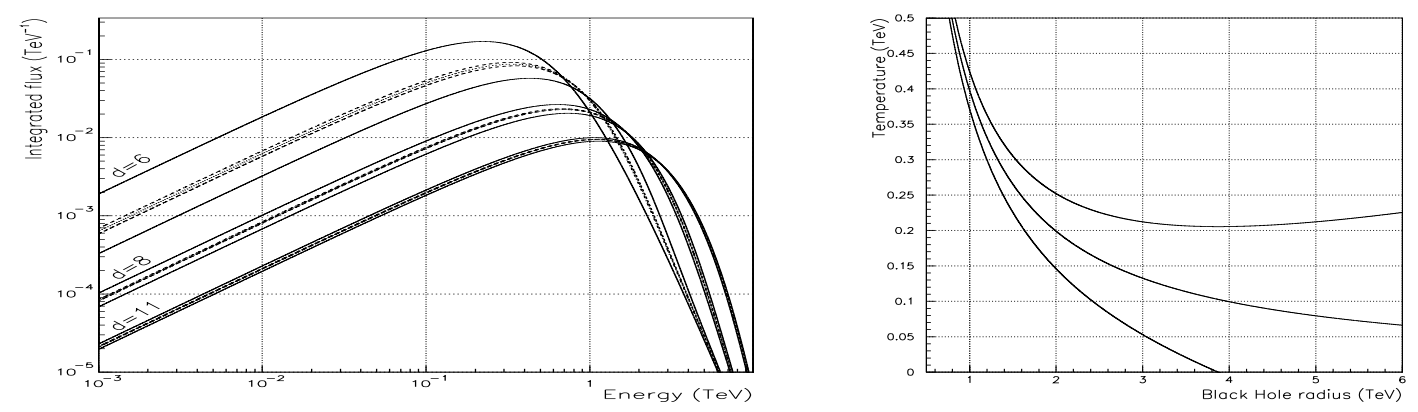

Figure 3: Left : Integrated flux (bosons) for $M_{d}=1 \mathrm{TeV}$ and $d=\{6,8,11\}$, from top to bottom $\Lambda=$ $\{-1,-0.1,0,0.1,1\} \mathrm{TeV}^{2}$. Right : Temperature of a $5 \mathrm{TeV}$ black hole in de-Sitter (bottom), anti-de-Sitter (top) and flat spacetimes for $d=8$ and $|\Lambda|=1 \mathrm{TeV}^{2}$.

Table 1 gives our estimates of the minimum required value of the cosmological constant to produce measurable effects. It should, of course, be kept in mind that this remains quite a theoretical approach as the associated size of the horizon is very small.

Figure 4 shows the results of the Monte-Carlo simulation of the evaporation process and underlines the degeneracies that could be broken by a detailed study of the greybody factors. As 
Table 1: Minimun cosmological constant that can be seen at the LHC with $100 \mathrm{fb}^{-1}$. In each case : dS, AdS, thermalized emission, instantaneous emission, ideal case (upper line), strong inelasticity and detector resolution taken into account (lower line). Only the extreme cases ( $\mathrm{d}=6$ and $\mathrm{d}=11$ are displayed).

\begin{tabular}{lcccc}
\hline \hline$|\Lambda|_{\text {min }}$ in $\mathrm{TeV}^{2}$ & \multicolumn{2}{c}{ Thermalized evaporation } & \multicolumn{2}{c}{ Sudden evaporation } \\
& $\mathrm{d} S$ & $\mathrm{AdS}$ & $\mathrm{dS}$ & $\mathrm{AdS}$ \\
\hline$d=6$ & 0.02 & 0.02 & 0.012 & 0.008 \\
& 0.52 & 0.44 & 0.30 & 0.22 \\
\multirow{3}{*}{$d=11$} & 0.18 & 0.18 & 0.14 & 0.12 \\
& 4.5 & 3.6 & 3.2 & 2.9 \\
\hline \hline
\end{tabular}
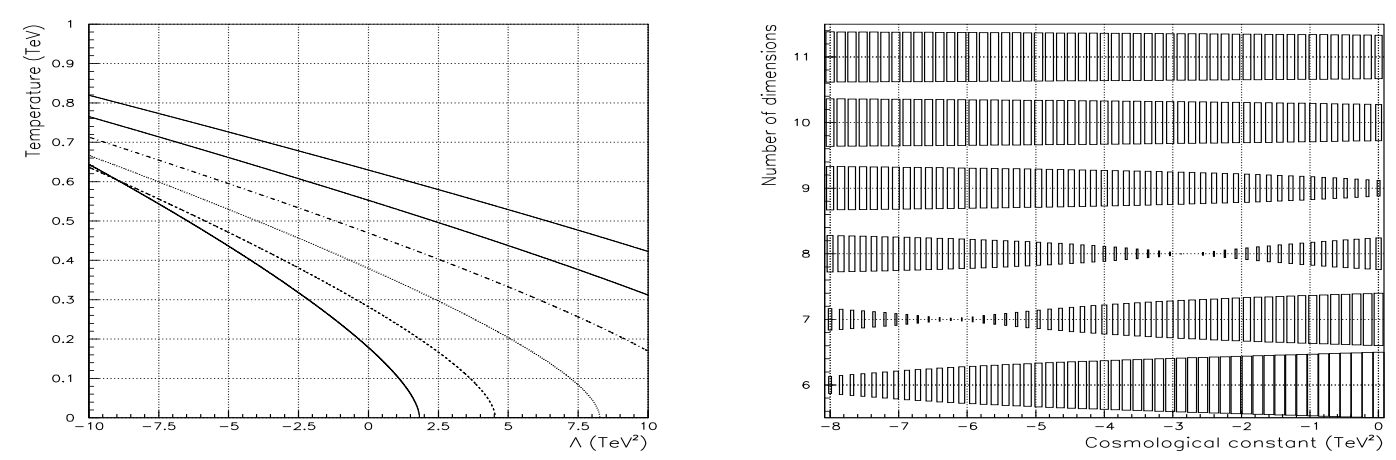

Figure 4: Left : temperature of a $5 \mathrm{TeV}$ black hole as a function of $\Lambda$ ( $d=6$ to 11 from bottom to top). Right : reduced $\chi^{2}$ for in put parameters : $d=8$ and $\Lambda=-2.5 \mathrm{TeV}^{2}$.

an example, for input parameters $d=8$ and $\Lambda=-2.5 \mathrm{TeV}^{2}$, the statistical analysis leads to $-4.8<\frac{\Lambda}{1 \mathrm{TeV}^{2}}<-0.8$ if the dimensionality is assumed to be otherwise measured. This opens exciting possibilities for experimental probes of a $d$-dimensional cosmological constant in the bulk.

\section{References}

[1] P. Kanti, Int. J. Mod. Phys. A 19 (2004) 4899

[2] P. Kanti, I. Olasagasti \& K. Tamvakis, Phys. Rev. D 66 (2002) 104026; P. Kanti \& J. March-Russell, Phys. Rev. D 67 (2003) 104019; C.M. Harris \& P. Kanti, JHEP 0310 (2003) 014; P. Kanti, J. March-Russell, Phys. Rev. D 66 (2002) 024023

[3] G. Duffy, C. Harris, P. Kanti \& E. Winstanley, hep-th/0507274

[4] J. Grain, A. Barrau \& P. Kanti, accepted by Phys. Rev. D, hep-th/0509128; A. Barrau, J. Grain \& S.O. Alexeyev, Phys. Lett. B 584 (2004) 114

[5] P. Kanti, J. Grain \& A. Barrau, Phys. Rev. D 71 (2005) 104002

[6] J. Grain, P. Kanti \& A. Barrau, in preparation

[7] T. Banks \& W. Fischler, hep-th/9906038; S. Dimopoulos and G. Landsberg, Phys. Rev. Lett. 87 (2001) 161602; S. B. Giddings and S. Thomas, Phys. Rev. D 65 (2002) 056010

[8] N. Arkani-Hamed, S. Dimopoulos \& G. R. Dvali, Phys. Lett. B 429 (1998) 263 